Bull. Austral. Math. Soc.

VOL. 65 (2002) [129-135]

\title{
SOME WEIGHTED ESTIMATES FOR IMAGINARY POWERS OF LAPLACE OPERATORS
}

\author{
Hendra Gunawan
}

\begin{abstract}
We study the boundedness of singular integral operators that are imaginary powers of the Laplace operator in $\mathbf{R}^{n}$, especially from weighted Hardy spaces $H_{w}^{p}\left(\mathbf{R}^{n}\right)$ to weighted Lebesgue spaces $L_{w}^{p}\left(\mathbf{R}^{n}\right)$ where $0<p \leqslant 1$. In particular, we prove some $H_{w}^{p}-L_{w}^{p}$ estimates for these operators when $0<p \leqslant 1$ and $w$ is in the Muckenhoupt's class $A_{q}$, for some $q>1$.
\end{abstract}

\section{INTRODUCTION}

We shall study a class of singular integral operators that are imaginary powers of the Laplace operator in $\mathbf{R}^{n}$. But first let us review some basic properties of singular integral operators in general.

It is well-known that every singular integral operator $T$ defined on $\mathcal{S}\left(\mathbf{R}^{n}\right)$ by

$$
T f=K * f
$$

where $K$ is a tempered distribution on $\mathbf{R}^{n}$ with $\widehat{K} \in L^{\infty}\left(\mathbf{R}^{n}\right)$, extends to a bounded operator on $L^{2}\left(\mathbf{R}^{n}\right)$. Provided that the kernel $K$ is locally integrable away from 0 and satisfies the Hörmander condition

$$
\int_{|x|>2|y|}|K(x-y)-K(x)| d x \leqslant C_{K}
$$

such an operator will also extend to a bounded operator on $L^{p}\left(\mathbf{R}^{n}\right)$ for $1<p<\infty$. For $p=1$ and $p=\infty$, weaker results are available.

Moreover, for $p=1$, one may also show that $T$ extends to a bounded operator from $H^{1}\left(\mathbf{R}^{n}\right)$ to $L^{1}\left(\mathbf{R}^{n}\right)$. In fact, with some extra conditions on $K$, the operator $T$ can be extended to a bounded operator from $H^{p}\left(\mathbf{R}^{n}\right)$ to $L^{p}\left(\mathbf{R}^{n}\right)$ for $0<p \leqslant 1$. Here, for each $0<p \leqslant 1, H^{p}\left(\mathbf{R}^{n}\right)$ denotes the Hardy space, whose members can be written as $\sum_{j} \lambda_{j} a_{j}$

\section{Received 27th June, 2001}

This note was written during a visit to the School of Mathematics, UNSW, Sydney, in 2000/2001, and was supported by an Australia-Indonesia Merdeka Fellowship funded by the Australian Government through the Department of Education, Training and Youth Affairs and promoted through Australia Education International. The author thanks Professor M. Cowling for his useful suggestions.

Copyright Clearance Centre, Inc. Serial-fee code: 0004-9727/02 \$A2.00+0.00. 
where the $a_{j}$ 's are $p$-atoms and $\lambda_{j}$ 's are real numbers such that $\sum_{j}\left|\lambda_{j}\right|^{p}<C\|f\|_{H^{p}}^{p}$. (A $p$-atom in $\mathbf{R}^{n}$ is a function $a$ supported in a finite cube $Q \subseteq \mathbf{R}^{n}$ such that $\|a\|_{\infty} \leqslant|Q|^{-1 / p}$ and $\int_{\mathbf{R}^{n}} a(x) x^{\alpha} d x=0$ for every multi-index $\alpha=\left(\alpha_{1}, \ldots, \alpha_{n}\right)$ with $|\alpha|=\alpha_{1}+\cdots+\alpha_{n}$ $\leqslant n(1 / p-1)$.) See [3, Chapters II and III].

Now let $w$ be a nonnegative measurable function or a weight on $\mathbf{R}^{n}$ and $L_{w}^{p}\left(\mathbf{R}^{n}\right)$ be the space of all functions $f: \mathbf{R}^{n} \rightarrow \mathbf{C}$ for which $\|f\|_{L_{w}^{p}}=\left[\int_{\mathbf{R}^{n}}|f(x)|^{p} w(x) d x\right]^{1 / p}<\infty$. Then one may show that $T$ extends to a bounded operator on $L_{w}^{p}\left(\mathbf{R}^{n}\right)$ for $1<p<\infty$ provided that $w \in A_{p}$, that is, $w$ satisfies the $A_{p}$ condition

$$
\left[\frac{1}{|Q|} \int_{Q} w(x) d x\right]\left[\frac{1}{|Q|} \int_{Q} w(x)^{-1 /(p-1)} d x\right]^{p-1} \leqslant C
$$

for all cubes $Q$ in $\mathbf{R}^{n}$. (For example, $|\cdot|^{a} \in A_{p}, 1<p<\infty$, if and only if $-n<a<$ $n(p-1)$.) As in the unweighted case, there is also a weaker result for $p=1$ and $w \in A_{1}$, satisfying the $A_{1}$ condition

$$
\frac{1}{|Q|} \int_{Q} w(x) d x \leqslant C w(y), \quad \text { almost everywhere } y \in Q,
$$

for all cubes $Q$ in $\mathbf{R}^{n}$, which can be viewed as the limit of $A_{p}$ conditions for $p \rightarrow 1^{+}$. (For example, $|\cdot|^{a} \in A_{1}$ if and only if $-n<a \leqslant 0$.) See [3, Chapter IV].

In this note, we shall study the boundedness of singular integral operators that are imaginary powers of the Laplace operator in $\mathbf{R}^{n}$, especially from $H_{w}^{p}\left(\mathbf{R}^{n}\right)$ to $L_{w}^{p}\left(\mathbf{R}^{n}\right)$ where $0<p \leqslant 1$. Here $H_{w}^{p}\left(\mathbf{R}^{n}\right)$ denotes the weighted Hardy space, defined just as $H^{p}\left(\mathbf{R}^{n}\right)$ but with measure $w(x) d x$ replacing the usual Lebesgue measure $d x$. These operators were studied by Muckenhoupt [6] and used by Cowling and Mauceri [1] to prove the boundedness of Stein's spherical maximal operator [9]. What we are interested in here is how their norms, especially from $H_{w}^{p}\left(\mathbf{R}^{n}\right)$ to $L_{w}^{p}\left(\mathbf{R}^{n}\right)$ where $0<p \leqslant 1$, actually depends on the imaginary power.

Recent works indicate that the study of imaginary powers of operators in general have some applications in the theory of spectral multipliers. See, for example, [2] and $[7]$.

\section{Main Results}

For each $u \in \mathbf{R} \backslash\{0\}$, let $K_{u}$ be the tempered distribution on $\mathbf{R}^{n}$ such that $\widehat{K_{u}}(\xi)=|\xi|^{-i u}$. Here $\widehat{K_{u}}$ is defined via $\left\langle\widehat{K_{u}}, f\right\rangle=\left\langle K_{u}, \widehat{f}\right\rangle \forall f \in \mathcal{S}\left(\mathbf{R}^{n}\right)$, with $\hat{f}(\xi)=\int_{\mathbf{R}^{n}} f(x) e^{2 \pi i x \cdot \xi} d x$ being the usual Fourier transform in $\mathbf{R}^{n}$. Explicitly, $K_{u}$ may be given by

$$
K_{u}(x)=C(u)|x|^{-n+i u}
$$


where $C(u)=\pi^{-n / 2+i u} \Gamma(n-i u / 2) / \Gamma(i u / 2)$ (see [8, p.117]). Then define the singular integral operator $I_{u}$ on $\mathcal{S}\left(\mathbf{R}^{n}\right)$ by

$$
I_{u} f=K_{u} * f .
$$

By Plancherel's theorem, we see that $I_{u}$ extends to an isometry on $L^{2}\left(\mathbf{R}^{n}\right)$, and that

$$
\left(I_{u} f\right)^{\frown}(\xi)=|\xi|^{-i u} \hat{f}(\xi)=(2 \pi)^{i u}\left(\Delta^{-i u / 2} f\right)^{\frown}(\xi),
$$

that is, $I_{u}$ is an imaginary power of the Laplace operator $\Delta=-\sum_{j=1}^{n} \partial_{j}^{2}$.

To be able to say more about $I_{u}$, let us examine its kernel $K_{u}$. Clearly $K_{u}$ is locally integrable away from 0 and, since $C(u)=\mathrm{O}\left((1+|u|)^{n / 2}\right)$, we see that $K_{u}$ satisfies

(a) $\left|K_{u}(x)\right| \leqslant C(1+|u|)^{n / 2}|x|^{-n}, \quad x \neq 0$, and

(b) $\left|K_{u}(x-y)-K_{u}(x)\right| \leqslant C(1+|u|)^{n / 2+1}|y||x|^{-n-1}, \quad|x|>2|y|>0$,

whence (by interpolation)

(c) $\left|K_{u}(x-y)-K_{u}(x)\right| \leqslant C(1+|u|)^{n / 2+\delta}|y|^{\delta}|x|^{-n-\delta}, \quad|x|>2|y|>0$,

for any $0 \leqslant \delta \leqslant 1$. From (c), one may check that $K_{u}$ satisfies the Hörmander condition

$$
\int_{|x|>2|y|}\left|K_{u}(x-y)-K_{u}(x)\right| d x \leqslant C_{\delta}(1+|u|)^{n / 2+\delta}
$$

whenever $0<\delta \leqslant 1$. Hence, our operator $I_{u}$ extends to a bounded operator on $L^{p}\left(\mathbf{R}^{n}\right)$ for $1<p<\infty$, and also from $H^{p}\left(\mathbf{R}^{n}\right)$ to $L^{p}\left(\mathbf{R}^{n}\right)$ for $n /(n+1)<p \leqslant 1$. Indeed, one may verify that

$$
\left\|I_{u} f\right\|_{L^{p}} \leqslant C_{p, \delta}(1+|u|)^{|n / p-n / 2|+\delta}\|f\|_{L^{p}}, \quad f \in L^{p}\left(\mathbf{R}^{n}\right),
$$

for $1<p<\infty$, and

$$
\left\|I_{u} f\right\|_{L^{p}} \leqslant C_{p, \delta}(1+|u|)^{n / p-n / 2+\delta}\|f\|_{H^{p}}, \quad f \in H^{p}\left(\mathbf{R}^{n}\right),
$$

for $n /(n+1)<p \leqslant 1$ and $0<\delta \leqslant 1$. By observing that, for every $k \in \mathbf{N}=\{1,2,3, \ldots\}$, $K_{u}$ is of class $C^{k}$ away from the origin, and satisfies

(d) $\left|D^{\beta} K_{u}(x)\right| \leqslant C(1+|u|)^{n / 2+k}|x|^{-n-|\beta|}, \quad x \neq 0$,

for every multi-index $\beta$ with $|\beta| \leqslant k$, one can show that $I_{u}$ extends to a bounded operator from $H^{p}\left(\mathbf{R}^{n}\right)$ to $L^{p}\left(\mathbf{R}^{n}\right)$ for $n /(n+k)<p \leqslant 1$, and hence for every $0<p \leqslant 1$ (see [3, pp.320-322]).

2.1. UNWEIGHTED $H^{p}-L^{p}$ ESTIMATES. As recently shown in [5], we can actually get rid of $\delta$ in the $H^{1}-L^{1}$ estimate for $I_{u}$ (and hence, by interpolation with the $L^{2}$ result and duality arguments, we can also get rid of $\delta$ in the $L^{p}-L^{p}$ estimate for $1<p<\infty$ ). This is the best we can achieve in the sense that we cannot have the exponent of $(1+|u|)$ less than $|n / p-n / 2|$. See also [7] for similar results.

The following theorem states that the same is also true for $0<p \leqslant 1$. 
ThEOREM 1. The $H^{p}-L^{p}$ inequality

$$
\left\|I_{u} f\right\|_{L^{p}} \leqslant C_{p}(1+|u|)^{n / p-n / 2}\|f\|_{H^{p}}, \quad f \in H^{p}\left(\mathbf{R}^{n}\right)
$$

holds for $0<p \leqslant 1$.

Proof: Suppose first that $n /(n+1)<p \leqslant 1$. By the atomic decomposition, it suffices to show that

$$
\left\|I_{u} a\right\|_{L^{p}} \leqslant C(1+|u|)^{n / p-n / 2}
$$

for any $p$-atom $a$. So, let $a$ be a $p$-atom, supported in a cube $Q$, such that $\|a\|_{\infty} \leqslant|Q|^{-1 / p}$ and $\int_{\mathbf{R}^{n}} a(y) d y=0$, and assume that $|u|>2$ (so that (b) holds for $|x|>|u||y|>0$ ). By translation-invariance, we may assume that $Q$ is centred at the origin, say $Q=[-R, R]^{n}$. Now, to estimate $\left\|I_{u} a\right\|_{L^{p}}$, write

$$
\int_{\mathbf{R}^{n}}\left|I_{u} a(x)\right|^{p} d x=\int_{|x|<|u| R}\left|I_{u} a(x)\right|^{p} d x+\int_{|x|>|u| R}\left|I_{u} a(x)\right|^{p} d x=\mathrm{I}+\text { II. }
$$

(Note the difference from the usual trick: instead of splitting the integral at $2 R$, we split it at $|u| R$, just as in [5].) For the first integral, we use the fact that $I_{u}$ is an isometry on $L^{2}\left(\mathbf{R}^{n}\right)$ and apply the Cauchy-Schwarz inequality to get

$$
I \leqslant\left[\int_{|x|<|u| R} d x\right]^{1-p / 2}\left[\int_{|x|<|u| R}\left|I_{u} a(x)\right|^{2} d x\right]^{p / 2} \leqslant(|u| R)^{n-n p / 2}\|a\|_{2} \leqslant|u|^{n-n p / 2}
$$

For the second integral, we first observe that by using (b) we have

$$
\begin{aligned}
\left|I_{u} a(x)\right| & =\left|\int_{|y|<R} K_{u}(x-y) a(y) d y\right| \\
& =\left|\int_{|y|<R}\left[K_{u}(x-y)-K_{u}(x)\right] a(y) d y\right| \\
& \leqslant \int_{|y|<R}\left|K_{u}(x-y)-K_{u}(x)\right||a(y)| d y \\
& \leqslant C(1+|u|)^{n / 2+1}|x|^{-n-1} \int_{|y|<R}|y||a(y)| d y \\
& \leqslant C(1+|u|)^{n / 2+1} R^{n+1-n / p}|x|^{-n-1},
\end{aligned}
$$

whenever $|x|>|u| R$. Hence, since $p>n /(n+1)$, we get

$$
\mathrm{II} \leqslant C^{p}(1+|u|)^{n p / 2+p} R^{n p+p-n} \int_{|x|>|u| R}|x|^{-n p-p} d x \leqslant C^{p}(1+|u|)^{n-n p / 2} .
$$

Combining with the previous estimate and then taking the $p$-th root, we obtain

$$
\left\|I_{u} a\right\|_{L^{p}} \leqslant C(1+|u|)^{n / p-n / 2}
$$


as desired.

Suppose now that $n /(n+k)<p \leqslant n /(n+k-1)$ for some $k \in \mathbf{N}$. Take a $p$-atom $a$ which is supported in $Q=[-R, R]^{n}$. We wish to show that

$$
\left\|I_{u} a\right\|_{L^{p}} \leqslant C(1+|u|)^{n / p-n / 2} .
$$

For this, we split again the integral at $|u| R$, with $|u|>2$. The estimate for the first integral will be exactly the same as before. For the second integral, we use the fact that $K_{u}$ is of class $C^{k}$ away from the origin and satisfies (d). We subtract from $K_{u}(x-y)$ the Taylor polynomial of $K_{u}$ at $x$ of degree $[n(1 / p-1)]=k-1$, to obtain

$$
\left|I_{u} a(x)\right| \leqslant C(1+|u|)^{n / 2+k} R^{n+k-n / p}|x|^{-n-k}, \quad|x|>|u| R .
$$

The estimate for the second integral will then follow immediately from this.

2.2. Weighted $H^{p}-L^{p}$ estimates. As for the unweighted case, one may easily verify that the weighted inequality

$$
\left\|I_{u} f\right\|_{L_{w}^{p}} \leqslant C_{p, w, \delta}(1+|u|)^{n / 2+\delta}\|f\|_{L_{w}^{p}}, \quad f \in L_{w}^{p}\left(\mathbf{R}^{n}\right),
$$

holds whenever $w \in A_{p}, 1<p<\infty$, and $\delta>0$ sufficiently small. The proof reduces to establishing the pointwise estimate

$$
\left(I_{u} f\right)^{\#}(x) \leqslant C_{q, \delta}(1+|u|)^{n / 2+\delta}\left[M\left(|f|^{q}\right)\right]^{1 / q}(x)
$$

for some $q>1$ such that $w \in A_{p / q}$. (Here $f \mapsto f^{\#}$ denotes the sharp maximal operator (see [10, p.146]), while $M$ is the standard Hardy-Littlewood maximal operator.) See [3, p.411], for why, and [10, pp.157-158], for how. See also [4] for an alternative proof. Further, as in the unweighted case, we can also get rid of $\delta$ here to obtain the sharp estimate (see [5]).

We shall now show that our operator $I_{u}$ can also be extended to a bounded operator from $H_{w}^{p}\left(\mathbf{R}^{n}\right)$ to $L_{w}^{p}\left(\mathbf{R}^{n}\right)$ where $0<p \leqslant 1$ and $w \in A_{q}$, for some $q>1$. More precisely, we have the following result.

THEOREM 2. Let $0<p \leqslant 1$. Suppose that $n /(n+k)<p \leqslant n /(n+k-1)$ for some $k \in \mathbf{N}$ and let $0<\varepsilon<n+k-n / p$. Then, the inequality

$$
\left\|I_{u} f\right\|_{L_{w}^{p}} \leqslant C_{p, w, \varepsilon}(1+|u|)^{n / p-n / 2+\varepsilon}\|f\|_{H_{w}^{p}}, \quad f \in H_{w}^{p}\left(\mathbf{R}^{n}\right),
$$

holds whenever $w \in A_{1+\varepsilon p / n}$.

Proof: We shall only prove the case where $k=1$. As usual, we shall use the atomic decomposition, which will reduce our task to showing that

$$
\left\|I_{u} a\right\|_{L_{w}^{p}} \leqslant C_{p, w, \varepsilon}(1+|u|)^{n / p-n / 2+\varepsilon}
$$


for any $p$-atom $a$ with respect to $w \in A_{q}$, where $q=1+\varepsilon p / n$. Let $a$ be a $p$-atom with respect to $w$, supported in a finite cube $Q$, such that $\|a\|_{\infty} \leqslant w(Q)^{-1 / p}$ and $\int_{\mathbf{R}^{\mathbf{n}}} a(y) w(y) d y=0$, and assume that $|u|>2$. We may also assume that $Q$ is centred at the origin, say $Q=[-R, R]^{n}$. Now write

$$
\int_{\mathbf{R}^{n}}\left|I_{u} a(x)\right|^{p} w(x) d x=\int_{|x| \leqslant|u| R}\left|I_{u} a(x)\right|^{p} w(x) d x+\int_{|x|>|u| R}\left|I_{u} a(x)\right|^{p} w(x) d x=\mathrm{I}+\mathrm{II} .
$$

To estimate I, we use Hölder's inequality and the fact that $I_{u}$ is bounded on $L_{w}^{q}\left(\mathbf{R}^{n}\right)$, with norm $\leqslant C_{q, w}(1+|u|)^{n / 2}$. Precisely, we have

$$
\begin{aligned}
I & \leqslant\left[\int_{|x| \leqslant|u| R} w(x) d x\right]^{1-p / q}\left[\int_{|x| \leqslant|u| R}\left|I_{u} a(x)\right|^{q} w(x) d x\right]^{p / q} \\
& \leqslant C_{q, w}^{p} w\left(Q^{|u|}\right)^{1-p / q}(1+|u|)^{n p / 2}\|a\|_{q, w}^{p} \\
& \leqslant C_{p, q, w}(1+|u|)^{n q-n p / 2}\|a\|_{q, w}^{p} w(Q)^{1-p / q}
\end{aligned}
$$

by [3, Lemma 2.2, p.396] (applied to $Q^{|u|}$, the $|u|$-dilate of $Q$ ). But

$$
\|a\|_{q, w}^{q}=\int_{\mathbf{R}^{n}}|a(x)|^{q} w(x) d x \leqslant \int_{Q} w(Q)^{-q / p} w(x) d x=w(Q)^{1-q / p},
$$

and so $\|a\|_{q, w}^{p} \leqslant w(Q)^{p / q-1}$, whence

$$
\mathrm{I} \leqslant C_{p, q, w}(1+|u|)^{n q-n p / 2}
$$

To estimate II, we first observe that

$$
\left|I_{u} a(x)\right| \leqslant C(1+|u|)^{n / 2+1} R^{n+1} w(Q)^{-1 / p}|x|^{-n-1},
$$

so that

$$
\text { II } \leqslant C(1+|u|)^{n p / 2+p} R^{n p+p} w(Q)^{-1} \int_{|x|>|u| R} \frac{w(x)}{|x|^{n p+p}} d x .
$$

But, since $q<p(n+1) / n$, we have

$$
\begin{aligned}
\int_{|x|>|u| R} \frac{w(x)}{|x|^{n p+p}} d x & =\sum_{j=1}^{\infty} \int_{2^{j-1}|u| R<|x| \leqslant 2 j|u| R} \frac{w(x)}{|x|^{n p+p}} d x \\
& \leqslant C \sum_{j=1}^{\infty}\left(2^{j}|u| R\right)^{-n p-p} w\left(Q^{2^{j}|u|}\right) \\
& \leqslant C(1+|u|)^{n q-n p-p} R^{-n p-p} w(Q) \sum_{j=1}^{\infty} 2^{j(n q-n p-p)} \\
& <C_{p}(1+|u|)^{n q-n p-p} R^{-n p-p} w(Q)
\end{aligned}
$$


and hence

$$
\mathrm{II} \leqslant C_{p}(1+|u|)^{n q-n p / 2} .
$$

Combining the two estimates, we get

$$
\int_{\mathbf{R}^{n}}\left|I_{u} a(x)\right|^{p} w(x) d x \leqslant C_{p, q, w}(1+|u|)^{n q-n p / 2} .
$$

Finally, substituting $q=1+\varepsilon p / n$ and then taking the $p$-th root, we obtain

$$
\left\|I_{u} a\right\|_{L_{w}^{p}} \leqslant C_{p, w, \varepsilon}(1+|u|)^{n / p-n / 2+\varepsilon} .
$$

This completes the proof.

Remark. Notice that as $\varepsilon$ tends to 0 , the exponent of $(1+|u|)$ tends to $n / p-n / 2$ and the set of weights $w$ for which the inequality holds tends to the Muckenhoupt's class $A_{1}$. However, we do not know whether the estimate

$$
\left\|I_{u} f\right\|_{L_{w}^{p}} \leqslant C_{p, w}(1+|u|)^{n / p-n / 2}\|f\|_{H_{w}^{p}, \quad f \in H_{w}^{p}\left(\mathbf{R}^{n}\right),}
$$

holds for $0<p \leqslant 1$ and $w \in A_{1}$.

\section{REFERENCES}

[1] M. Cowling and G. Mauceri, 'On maximal functions', Rend. Sem. Mat. Fis. Milano 49 (1979), 79-87.

[2] M. Cowling and A. Sikora, 'A spectral multiplier theorem for a sublaplacian on SU(2)', Math. Z. 238 (2001), 1-36.

[3] J. García-Cuerva and J.-L. Rubio de Francia, Weighted norm inequalities and related topics, North-Holland Mathematical Studies 116 (North-Holland, Amsterdam, 1985).

[4] H. Gunawan, 'On weighted estimates for Stein's maximal function', Bull. Austral. Math. Soc. 54 (1996), 35-39.

[5] H. Gunawan and J. Wright, 'Weighted estimates for some singular integrals', (preprint 2001).

[6] B. Muckenhoupt, 'On certain singular integrals', Pacific J. Math. 10 (1960), 239-261.

[7] A. Sikora and J. Wright, 'Imaginary powers of Laplace operators', Proc. Amer. Math. Soc. 129 (2001), 1745-1754.

[8] E.M. Stein, Singular integrals and differentiability properties of functions, Princeton Mathematical Series 30 (Princeton Univ. Press, Princeton N.J., 1970).

[9] E.M. Stein, 'Maximal functions: spherical means', Proc. Nat. Acad. Sci. USA 73 (1976), 2174-2175.

[10] E.M. Stein, Harmonic analysis: Real-variable methods, orthogonality, and oscillatory integrals, Princeton Mathematical Series 43 (Princeton Univ. Press, Princeton N.J., 1993).

Department of Mathematics

Bandung Institute of Technology

Bandung 40132

Indonesia

e-mail: hgunawan@dns.math.itb.ac.id 